\title{
Introduction of 6th Asian-Pacific Symposium on Radiochemistry (APSORC17)
}

\author{
Wansik Cha ${ }^{1} \cdot$ Jei-Won Yeon ${ }^{1} \cdot$ Kwang Yong Jee ${ }^{1}$
}

Published online: 8 May 2018

(C) Akadémiai Kiadó, Budapest, Hungary 2018

The Asia-Pacific Symposium on Radiochemistry 2017 (APSORC17) was the sixth of a series of quadrennial conferences started in 1997 in Kumamoto (Japan). APSORC has taken place at Fukuoka (Japan) in 2001, Beijing (China) in 2005, Napa (CA, USA) in 2009, and Kanazawa (Japan) in 2013. Since the announcement in 2013 of hosting the APSORC17 in Korea, our institution, Korea Atomic Energy Research Institute (KAERI), has taken a role as the main organizer in cooperation with Korea Nuclear Society (KNS). Over the past two decades, we have seen the growth of APSORC and noted that it has provided an excellent platform for the exchange of scientific knowledge in diverse fields in radiochemistry. In these areas KAERI also has played a central role in incubating and expanding radiochemistry in Korea, as it celebrates its 60th anniversary next year. Recently, we have invested greater efforts into resolving key issues such as nuclear non-proliferation and the safe disposal of high-level radioactive wastes originating from the progress and maturity of Korean nuclear energy production. In this respect, the local organizing committee (LOC) agreed to host APSORC17 under the theme "Radiochemistry for Peaceful Prosperity." As my colleagues and I expected, it became the first comprehensive international symposia covering various fields of radiochemistry in Korea. The conference was held in Jeju Island during 17-22 September 2017 with great success.

We selected 326 abstracts in total and about 370 persons registered for the conference. After being reviewed by the scientific committee, the abstracts were assigned for presentation including 6 plenary lectures, 40 invited lectures, and 140 oral and 140 poster presentations. There were 10 exhibitors who displayed their products in relation to radioanalytical techniques. At the conference 335

Kwang Yong Jee

nkyjee@kaeri.re.kr

1 Nuclear Chemistry Research Division, Korea Atomic Energy Research Institute, Daejeon 34057, Korea participants (including 15 accompanying persons) representing 21 countries around the world attended five plenary sessions, 39 oral sessions, and two poster sessions (Fig. 1). There was plenty of time for discussion between sessions and at social programs (Fig. 2).

In APSORC17 most traditional technical areas dealt with in the previous series of the conferences were covered as well as a special session of (1) Nuclear Non-proliferation. A total of twelve technical sessions were arranged, and the remaining were as follows: (2) Severe Accident \& Environmental Remediation Issues, (3) Nuclear Chemistry, (4) Nuclear Energy Chemistry, (5) Nuclear Analytical Techniques, (6) Separation Chemistry, (7) Actinide Chemistry, (8) Nuclear Forensics, (9) Environmental Radiochemistry and Radioecology, (10) Radiopharmaceutical Chemistry, (11) Application of Nuclear and Radiochemical Techniques, and (12) Education in Nuclear and Radiochemistry. In a special session arranged for the Hevesy Medal Award (HMA) Prof. Amares Chatt of the JRNC Board of the Hevesy Award announced the winner of HMA 2017: Prof. Pavel P. Povinec. He presented his award lecture on New ultra-sensitive radioanalytical technologies for new sciences following the medal award ceremony with Dr. Kwang Yong Jee, the chair of APSORC17 LOC.

The six invited speakers in plenary sessions held each morning made huge contributions to the success of this conference by providing comprehensive reviews and perspectives in different areas of radiochemistry. I would like to give my special thanks to the speakers for the following lectures: Actinide Chemistry relevant to the Long-term Safety of Nuclear Waste Disposal by Jae-Il Kim (former director of KIT-INE, Germany); Limitations on Containment of Nuclear Programs by Olli Heinonen (senior advisor of SNF-DD, USA); Superheavy Elements of the Mendeleev's Periodic Table: Present Status and Future by Sergey Dmitriev (director of FLNR-JINR, Russian Federation); Chemistry of the Heaviest Elements by Yuichiro Nagame (deputy director of ASRC-JAEA, Japan); New 


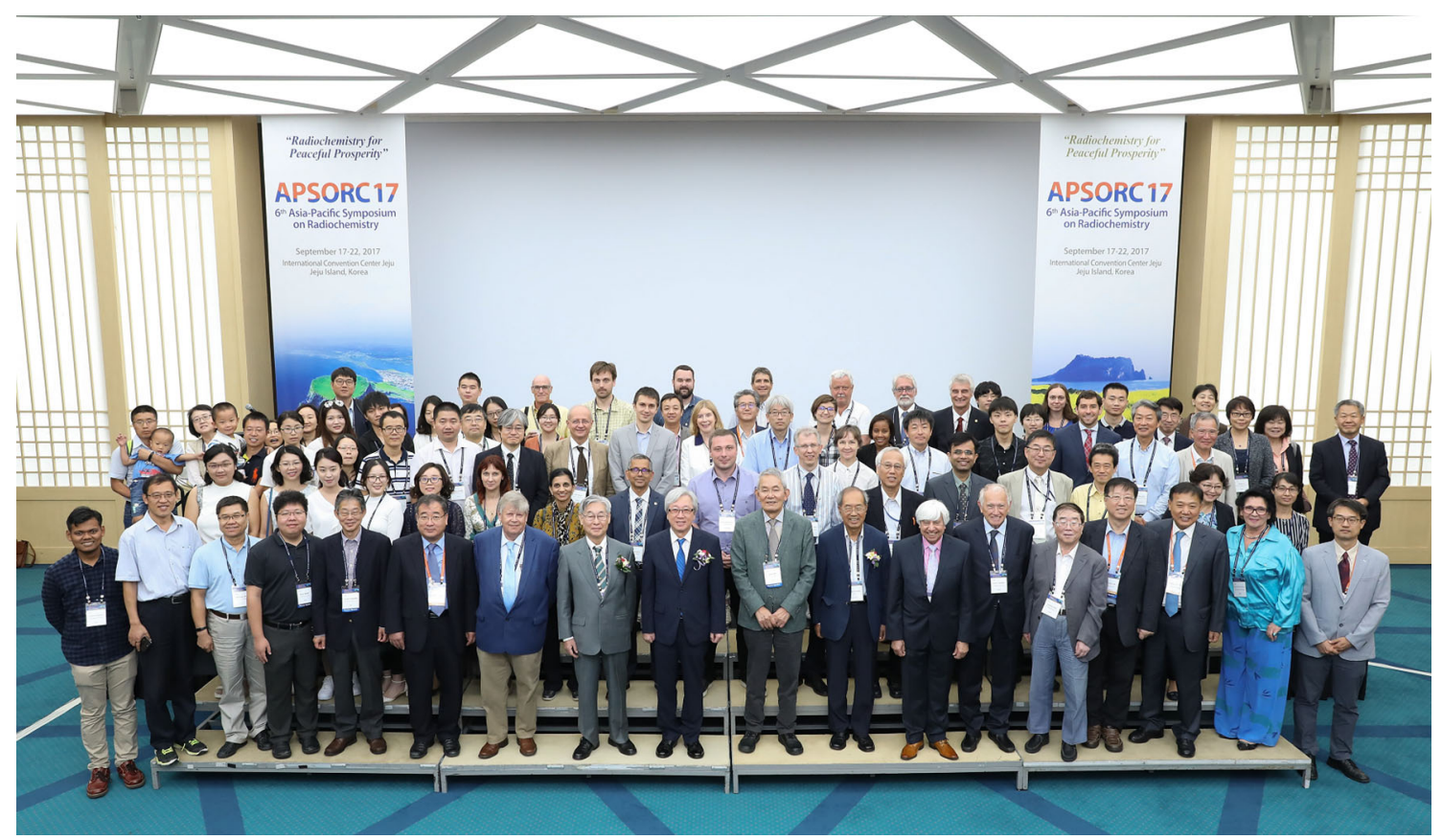

Fig. 1 Conference participants' group photo (APSORC17, Jeju Island)

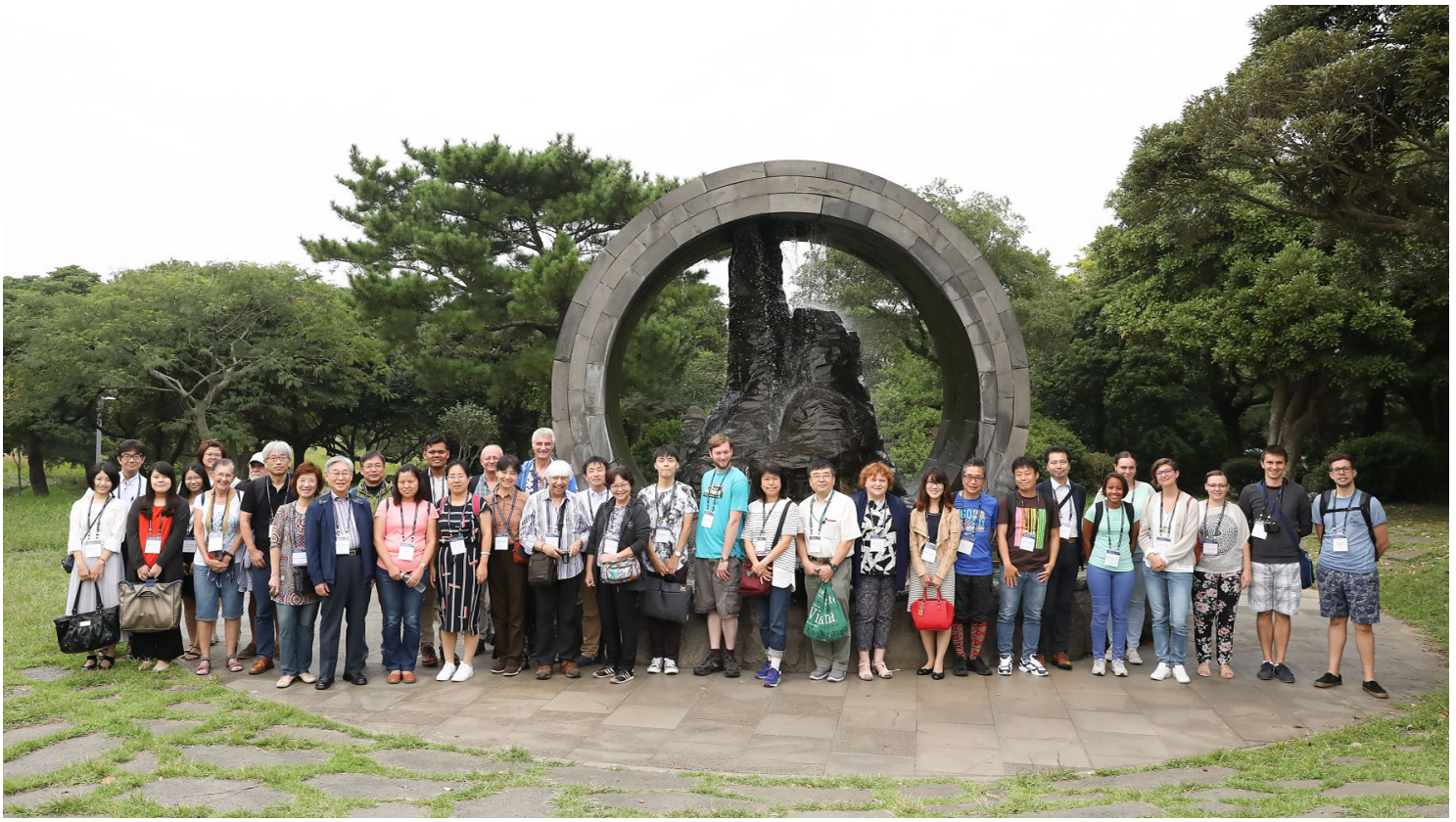

Fig. 2 Excursion group photo (APSORC17, Jeju Island)

Progress of Chemistry of Actinides and Fission Products by Shuao Wang (SRMP, RAD-X, Soochow University, China); and Development and Perspective of PET Radiopharmaceuticals by Dae Yoon Chi (Sogang University, Korea).

I know that the success of the conference ultimately depended on the many people who worked with us in the planning and organizing of this conference. In particular, we thank the International Advisory Committee (IOC), LOC, and Technical Program Committee, who contributed to arranging the scientific sessions and presenting invited talks.

The international organizing committee (IOC) consisted of the following members: Toshiaki Kishikawa (conference chair; Kumamoto University, Japan), Amares Chatt (ViceChair; Dalhousie University, Canada), Yuichiro Nagame 
(Vice-Chair; JAEA, Japan), Noriyuki Momoshima (secretary general; Kyushu University, Japan), Akihiko Yokoyama (undersecretary general; Kanazawa University, Japan), John W. Bennett (Australian Nuclear Science \& Technology Organization, Australia), Zhifang Chai (Chinese Academy of Sciences, China), Yuliang Zhao (National Center for Nanoscience \& Technology, China), Abdul Mutalib (National Nuclear Energy Agency, Indonesia), Mitsuru Ebihara (Tokyo Metropolitan University, Japan), Yong Hee Chung (Hallym University, Korea), Kyu-Seok Song and Jei-Won Yeon (KAERI, Korea), Sergey N. Dmitriev (Joint Institute for Nuclear Research, Russian Federation), Vladimir P. Kolotov (Vernadsky Institute of Geochemistry and Analytical Chemistry, Russian Federation), and Kanokporn Boonsirichai (Thailand Institute of Nuclear Technology, Thailand).

Grateful thanks are also expressed to the LOC members of APSORC17 and my colleagues in KAERI: Kwang Yong Jee (chair; KAERI), Jei-Won Yeon (secretary general; KAERI), Wansik Cha (secretary, KAERI), Dae Yoon Chi (Sogang University), Yong Hee Chung (Hallym University), Kwang-Sup Choi (KORAD), Eun-Hee Kim (Seoul National University), Kwang Pyo Kim (Kyungheee University), Byeoung-Kug Lee (KHNP), Yong Kyun Kim (Hanyang University), Jin-Hong Lee (Chungnam National University), Kil-Yong Lee (KIGAM), Chan-Soo Park (KBSI), Byung Gi Park (Soonchunhyang University), Tae
Soon Park (KRISS), Soon Hwan Son (KHNP-CRI), KyuSeok Song (KAERI), Jongsoon Song (Chosun University), Kook-Hyun Yu (Dongguk University), and Jong-Il Yun (KAIST). I wish to specially thank the company, The Plan, which was responsible for all logistical arrangements, and the governmental, institutional, and industrial sponsors in Korea for their financial support in various ways.

In this issue, 54 papers presented in the conference are published after the peer review process. For selection of papers, Prof. Jong-Il Yun (KAIST) managed the review process as a guest editor. I appreciate his efforts and contribution to in this special issue. Following this foreword are laudatory remarks on the Hevesy awardee by Prof. Amares Chatt and a review paper by Prof. Pavel P. Povinec on his activities. We would like to express our sincere thanks to Profs. Zsolt Révay (editor-in-chief of JRNC) and Amares Chatt (editor of JRNC), and Dr. Tibor G. Kocsor (managing editor of JRNC, Akadémiai Kiadó) for their support in timely publication of this special issue in JRNC.

Lastly, we thank all again for supporting us in organizing the conference, and for participation and coming to Jeju Island, Korea. I would like to encourage all readers to keep informed and to plan to attend APSORC 2021 to be held in Japan. 